\title{
REVIEW
}

\section{X-linked adrenoleukodystrophy: Diagnostic and follow-up system in Japan}

\author{
Nobuyuki Shimozawa ${ }^{1}$, Ayako Honda ${ }^{1}$, Naomi Kajiwara ${ }^{1}$, Sachi Kozawa ${ }^{1}$, Tomoko Nagase ${ }^{1}$, \\ Yasuhiko Takemoto ${ }^{1}$ and Yasuyuki Suzuki ${ }^{2}$
}

X-linked adrenoleukodystrophy (ALD) is an intractable neurodegenerative disease associated with the accumulation of very longchain saturated fatty acids (VLCFA) in tissues and body fluids. We have established a Japanese referral center for the diagnosis of ALD, using VLCFA measurements and mutation analysis of the $A B C D 1$ gene, and have identified 60 kinds of mutations in 69 Japanese ALD families, which included 38 missense mutations, 6 nonsense mutations, 8 frame-shift mutations, 3 amino acid deletions, 2 exon-skip mutations and 3 large deletions. A total of 24 kinds of mutations (40\%) were identified only in Japanese patients by referring to the current worldwide ALD mutation database. There was no clear correlation between these mutations and phenotypes of 81 male patients in these 69 families. About $12 \%$ of the individuals with ALD had de novo mutations by mutation analysis in the male probands and their mothers, which should be helpful data for genetic counseling. The only effective therapy for the cerebral form of ALD should be hematopoietic stem cell transplantation at the early stages of the cerebral symptoms, therefore, we performed presymptomatic diagnosis of ALD by extended familial screening of the probands with careful genetic counseling, and established a long follow-up system for these patients to prevent the progression of brain involvement and to monitor the adrenocortical insufficiency. Further elucidation of pathology in ALD, especially concerning the mechanisms of the onset of brain involvement, is expected.

Journal of Human Genetics (2011) 56, 106-109; doi:10.1038/jhg.2010.139; published online 11 November 2010

Keywords: adrenoleukodystrophy; diagnostic system; long-term follow-up; presymptomatic diagnosis; very long-chain fatty acids

\section{INTRODUCTION}

$\mathrm{X}$-linked adrenoleukodystrophy (ALD) is the most common peroxisomal disease, involving impaired $\beta$-oxidation of saturated very longchain fatty acids (VLCFA) resulting in the accumulation of VLCFA in tissues and plasma. The pathogenic gene for ALD, $A B C D 1$, has been mapped to Xq28, and the gene product, ALDP, is a peroxisomal membrane protein homologous to the ATP-binding cassette transporter superfamily of proteins, which can act as a transporter of VLCF acyl-CoA across the peroxisomal membrane. There are various clinical phenotypes such as the childhood cerebral form (CCALD), the adolescent cerebral form, the adult cerebral form, adrenomyeloneuropathy (AMN), the olivo-ponto-cerebellar form and Addison's disease only. ${ }^{1}$ CCALD is the most common phenotype and is characterized by progression of intellectual, psychic, visual and gait disturbances between the ages of 3 and 10. The prognosis of CCALD is generally very poor and patients were considered to die within few years, but good general care has improved the prognosis these days. Hematopoietic stem cell transplantation (HSCT) is currently the only curative approach, which can prevent the progression of brain involvement in the cerebral form of ALD, however, HSCT is only effective for patients in the early stages of cerebral symptoms. ${ }^{2}$ Therefore, we established a quick diagnostic system for ALD patients using combined measurements of VLCFA in the serum and mutation analysis of the ABCD1 gene. Nonetheless, even now only about one-third of the patients with CCALD have taken HSCT in Japan (data not shown), so presymptomatic diagnosis and early intervention are important for overcoming this intractable disease.

In this review, we introduce our diagnostic system and mutation range of Japanese patients with ALD, plus early intervention treatment and long-term follow-up for the presymptomatic ALD patients.

\section{DIAGNOSTIC SYSTEM AND MUTATION RANGE OF JAPANESE PATIENTS WITH ALD}

We analyze the VLCFA in serum or plasma from the patients suspected of ALD by clinical and brain magnetic resonance imaging (MRI) findings, using gas chromatography/mass spectrometry analysis. ${ }^{3}$ The patients are diagnosed by increases in VLCFA and we then proceed to molecular analysis of the $A B C D 1$ gene. The ratios of C24:0/ C22:0, C25:0/C22:0 and C26:0/C22:0 in male patients with ALD were significantly higher than control values, however, the ratios in some of the female carriers and control values overlapped (Figure 1). Moser et al. ${ }^{4}$ also reported that $85 \%$ of obligate heterozygotes had abnormally

${ }^{1}$ Division of Genomics Research, Life Science Research Center, Gifu University, Gifu, Japan and ${ }^{2}$ Medical Education Development Center, Gifu University School of Medicine, Gifu, Japan

Correspondence: Dr N Shimozawa, Division of Genomics Research, Life Science Research Center, Gifu University, Yanagido 1-1, Gifu 501-1193, Japan.

E-mail: nshim@gifu-u.ac.jp

Received 8 September 2010; revised 1 October 2010; accepted 13 October 2010; published online 11 November 2010 

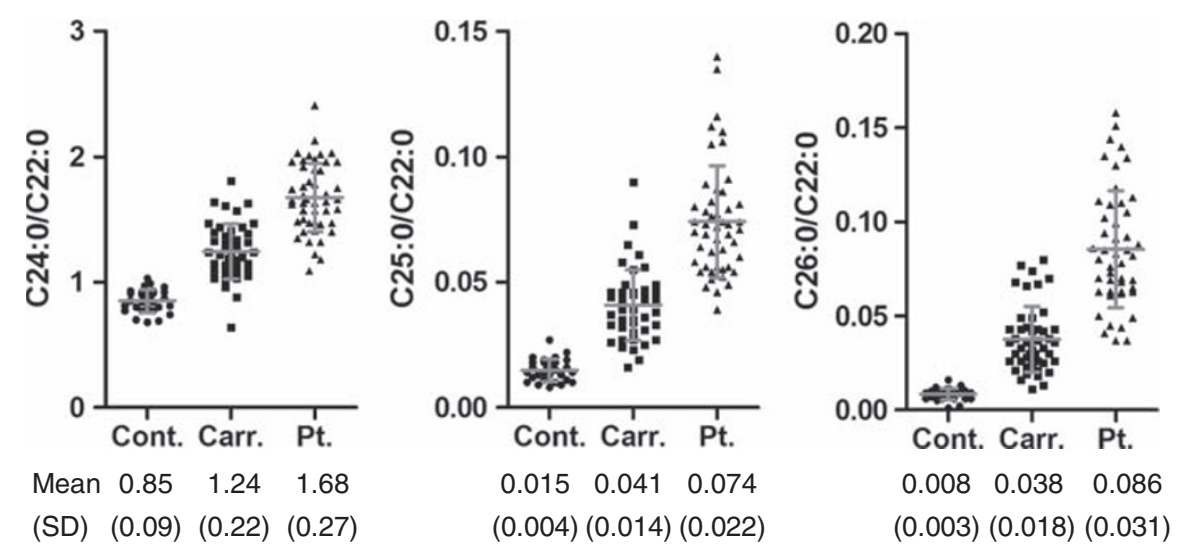

Figure 1 Plasma VLCFA levels in male patients, female carriers and controls. Values are given as mean (s.d.). Carr, female ALD carriers ( $n=46)$; Cont, controls ( $n=29)$; Pt, male ALD patients $(n=48)$; VLCFA: very long-chain fatty acids.

\section{ABCD1 mutations in 69 Japanese ALD families}

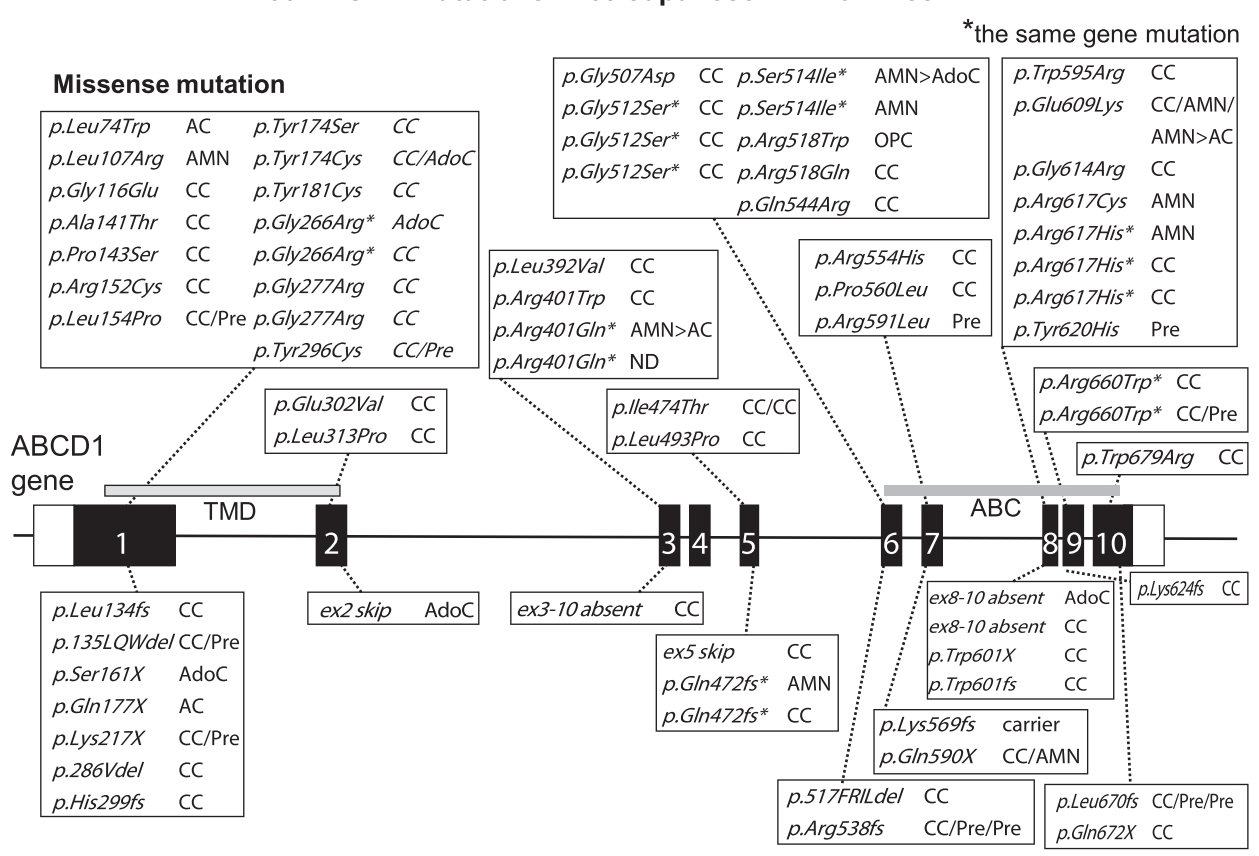

Nonsense / Frameshift (fs) / Amino acids del. / exon skip / large del.

Figure $260 A B C D 1$ mutations in 69 Japanese ALD families. Phenotypes were at the point of molecular analysis in time. ABC, ATP-binding cassette region; AC, adult cerebral form; AdoC, adolescent cerebral form; AMN, adrenomyeloneuropathy; CC, childhood cerebral form; ND, not determined; Pre, presymptomatic or asymptomatic; TMD, transmembrane domain; *, the same mutations were detected in two or three families.

high VLCFA levels, but also that a normal result does not exclude carrier status. Therefore, male ALD patients can be diagnosed only by increase in VLCFA, but female carriers should undergo molecular analysis of the $A B C D 1$ gene in order to get an accurate diagnosis.

Mutations of the $A B C D 1$ gene were analyzed with genetic counseling, by a modification of the method of Boehm et al., ${ }^{5}$ using genomic DNA extracted from the venous blood or cultured fibroblasts of the patients, which covered all coding regions and exon-intron junctions of the $A B C D 1$ gene. Among 69 Japanese probands with ALD (mainly CCALD) and their families, we identified 60 kinds of mutations, which included 38 missense mutations (63\%), 6 nonsense mutations (10\%), 8 frame shift mutations (13\%), 3 amino acid deletions (5\%), 2 exon-skip mutations (3\%) and 3 large deletions (5\%) (Figure 2). Although most probands with ALD identified by us had a unique gene mutation, 6 missense mutations (p.Gly266Arg, p.Arg401Gln, p.Gly512Ser, p.Ser514Ile, p.Arg617His and p.Arg660Trp) and 1 frame-shift mutation (p.Gln472fs) were detected in two or three families $\left({ }^{*}\right.$ in Figure 2$)$.

In the ALD Database (http://www.x-ald.nl), 1084 ABCD1 gene mutations have been updated and 533 kinds of non-recurrent mutations (49\%) were widely distributed over the $A B C D 1$ gene, which included $51 \%$ missense mutations, $11 \%$ nonsense mutations, $28 \%$ frame-shift mutations, $6 \%$ amino acid insertions/deletions and 3\% one or more exon deletions. The majority of missense mutations were located in two regions in the transmembrane domain and the ATP-binding cassette region in the ALDP protein (Figure 2), which means these domains should be important for the function of ALDP. The mutation range of Japanese ALD patients identified by us showed 
a tendency similar to the international database, however, concerning individual mutations, 24 of 60 kinds $(40 \%)$ were detected only in Japanese patients by referring to the current worldwide ALD mutation database.

Furthermore, our data demonstrated that in 33 families analyzed of the mutations in both the male probands and their mothers, four mothers had no mutations identified in the probands, which means about $12 \%$ of the individuals with ALD may have de novo mutations. In the ALD database, about $93 \%$ of index cases have inherited the mutations from one parent. Both data suggest that quite a few male probands might not inherit the disease-causing mutations from their mothers and that accurate carrier detection is necessary not only for further familial analysis but also for genetic counseling.

The phenotypes of 142 patients in 69 families analyzed by us, included 52 patients with childhood cerebral form, five with adolescent cerebral form, two with adult cerebral form, seven with AMN, three with AMN which had progressed to the adolescent or adult cerebral form $(\mathrm{AMN}>\mathrm{AdoC}$ or $\mathrm{AMN}>\mathrm{AC})$, one with the olivoponto-cerebellar form, 11 with presymptomatic form (Pre) and 60 with female carrier (Figure 2). There was no clear correlation between these mutations and the phenotypes in Japanese patients with ALD, as in previous reports. ${ }^{1}$ Even among the same families with the same mutations of $A B C D 1$, there was variability of phenotypes. At present, we have no means of predicting further clinical courses for presymptomatic ALD patients by analysis of family trees, genotypes, VLCFA values or even expression of the $A B C D 1$ gene and ALDP protein.

\section{HSCT AND PRESYMPTOMATIC DIAGNOSIS OF ALD}

HSCT is the only curative approach that can prevent the progression of brain involvement in the cerebral form of ALD to date; however, it is only effective for patients in the early stages of cerebral symptoms. ${ }^{2}$ Although we have established a quick diagnostic system for ALD in
Japan, only one-third of CCALD patients diagnosed after onset could undergo HSCT (data not shown) for several reasons. In the first place, for quite a few CCALD patients there was an interval between onset and brain MRI/VLCFA testing because they were initially diagnosed with attention-deficit/hyperactivity disorders, learning disability, hearing disturbance, exotropia and visual loss. In addition, it takes considerable time to prepare for HSCT, including searching for matched donors, and the HSCT procedure for patients with the advanced cerebral form could accelerate neurologic decline. Therefore, presymptomatic diagnosis by extended familial screening of probands should be valuable for early intervention and for preventing advanced cerebral symptoms. Furthermore, at least $70 \%$ of patients with CCALD and AMN have clinical and biochemical evidence of primary adrenocortical insufficiency (http://www.X-ald.nl), therefore, presymptomatic diagnosis should also be useful for preparing adrenal hormone therapy, which is necessary to save the lives of all ALD patients who have adrenal insufficiency.

Carrier detection with genetic counseling, which can be done by noting increased VLCFA in plasma, and mutation analysis of the $A B C D 1$ gene are very important to identify presymptomatic patients and other female carriers among their relatives. Elevated VLCFA levels confirm the diagnosis of carriers, however, there can be overlaps of the C24:0/C22:0, C25:0/C22:0 and C26:0/C22:0 ranges between healthy controls and at least $10 \%$ of the carriers (Figure 1). Furthermore, our data and others demonstrated about $10 \%$ of the probands were caused by spontaneous mutation of the $A B C D 1$ gene, which means that the mothers of these patients should not be carriers. For these reasons, mutation analysis of the $A B C D 1$ gene is necessary not only for carrier detection but also for confirming spontaneous mutation. In all, $32-48 \%$ of the female carriers of at least 20 years of age had some symptoms, which are mainly due to abnormalities in the spinal cord and nerves in the legs, similar to AMN. It appeared that with increasing age, the frequency of carriers that became symptomatic

\section{Follow-up flowchart for presymptomatic ALD}

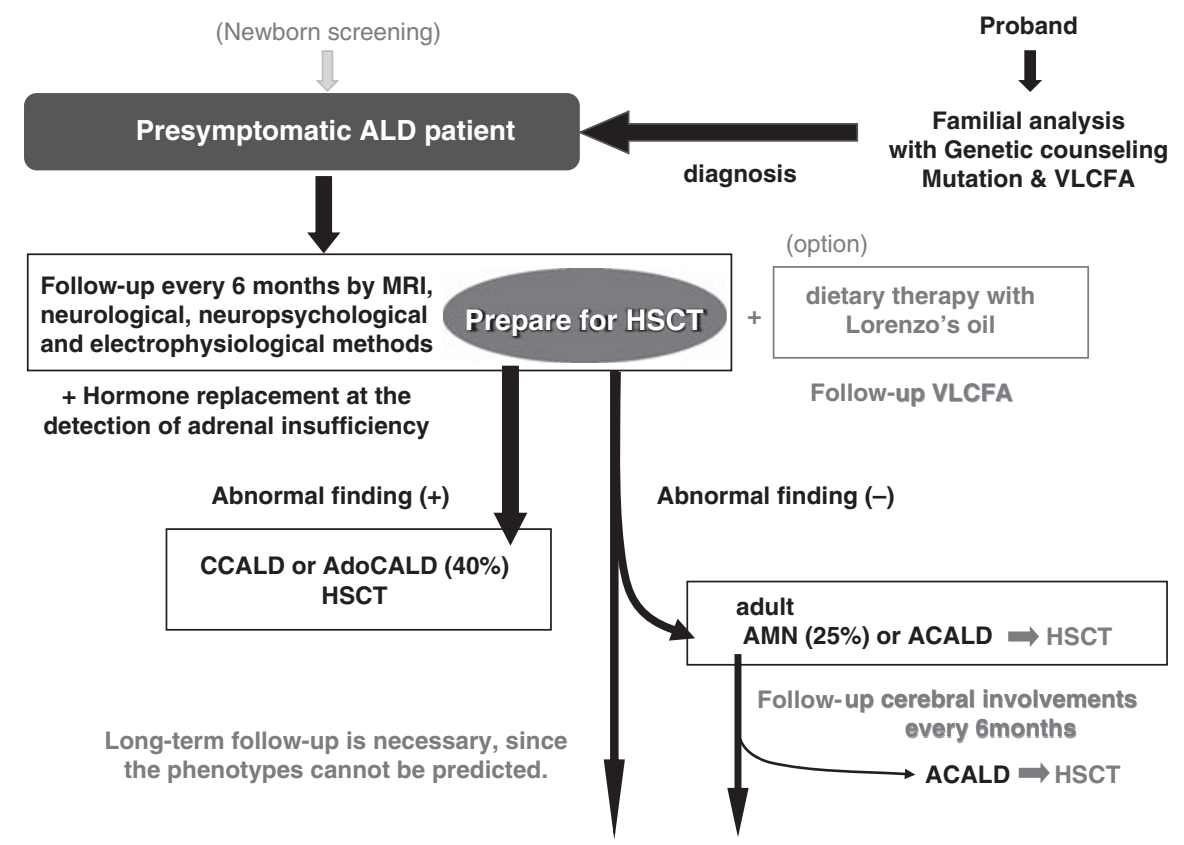

Figure 3 Follow-up flowchart for presymptomatic ALD. ACALD, adult cerebral form ALD; AdoCALD, adolescent cerebral form ALD; AMN, adrenomyeloneuropathy; CCALD, childhood cerebral form ALD; HSCT, hematopoietic stem cell transplantation; MRI, magnetic resonance imaging; VLCFA, very long-chain fatty acids. 
increased (http://www.x-ald.nl). Proper medication is often recommended for these carriers to reduce spasticity of the legs and pain in the lower back and hips. In future, newborn screening may be a potential method to discover presymptomatic male patients and female carriers widely. Hubbard et al. ${ }^{6}$ have started a pilot study of mass screening by the detection of 1-hexacosanoyl-2-lyso-sn-3-glycerophosphorylcholine (26:0-lyso-PC) using a combined liquid chromatography-tandem mass spectrometric method.

\section{EARLY INTERVENTION AND LONG-TERM FOLLOW-UP SYSTEM FOR PRESYMPTOMATIC ALD PATIENTS}

Presymptomatic male patients will progress to various phenotypes between 3 and 50 years old, and the phenotypes may be different from those of the probands. Therefore, it is important to give information to patients and family members by means of genetic counseling. After diagnosis of presymptomatic ALD male patients upto 3 years of age, for HSCT, follow-up every 6 months concerning subtle neuropsychological signs, brain MRI and electrophysiological investigation will be beneficial for such young boys. When any abnormalities are found, HSCT should be done as soon as possible (Figure 3). Lorenzo's oil is one of the options for these presymptomatic patients because Moser et al. reported that Lorenzo's oil therapy for asymptomatic ALD boys with normal brain MRI reduced the risk of developing MRI abnormalities, ${ }^{7}$ however, further study which verifies that effectiveness has not been reported. Additionally, it was reported that approximately $80 \%$ of asymptomatic boys with ALD had impaired adrenal function, ${ }^{8}$ therefore, all male patients should be monitored for adrenal insufficiency. In patients with morbid adrenal insufficiency, adrenal hormone replacement therapy is necessary and effective.

The establishment of a long-term follow-up system for these presymptomatic patients is necessary because we cannot predict suitable timing when HSCT should be done to get beneficial effects nor the subsequent clinical outcome. In our nationwide survey in Japan, about $30 \%$ of ALD occurred with cerebral symptoms before 10 years of age, whereas about $40 \%$ of patients had no obvious symptoms until 20 years of age. ${ }^{9}$ Furthermore, even in AMN patients, about half of these patients developed into adult cerebral ALD about 10 years after the onset, ${ }^{10}$ therefore, it is necessary to follow-up cerebral involvement every 6 months in AMN patients also (Figure 3).

Very recently, HSC gene therapy using a lentiviral vector has been reported to be successful in arresting the progression of CCALD in two patients at an early stage, ${ }^{11}$ however, the mechanisms of the therapeutic effect in this therapy still remain obscure, which may be because of replacement of the $A B C D 1$ gene, that of normal stem cells or other factors. Further studies concerning the modulation of VLCFA or the prevention of cerebral involvement should be accelerated for suffering patients and their families, as well as the identification of predictive factors for the onset of brain involvement as the keys to solving the problems.

\section{ACKNOWLEDGEMENTS}

This work was supported by a Grant-in-aid for Scientific Research (21591318) from the Japan Society for the Promotion of Science, and a Grant for Child Health Development and Research on Intractable Diseases from the Health and Labour Sciences Research Grants.

1 Moser, H. W., Smith, K. D., Watkins, P. A., Powers, J. \& Moser, A. B. X-linked adrenoleukodystrophy. in The Metabolic and Molecular Basis of Inherited Disease (eds Scriver, C. R., Beaudet, A. L., Sly, W. S., \& Valle, D.) 3257-3301 (McGraw-Hill, New York, 2001).

2 Peters, C., Charnas, L. R., Tan, Y., Ziegler, R. S., Shapiro, E. G., DeFor, T. et al. Cerebral X-linked adrenoleukodystrophy: the international hematopoietic cell transplantation experience from 1982 to 1999. Blood 104, 881-888 (2004).

3 Takemoto, Y., Suzuki, Y., Horibe, R., Shimozawa, N., Wanders, R. J. \& Kondo, N. Gas chromatography/mass spectrometry analysis of very long chain fatty acids, docosahexaenoic acid, phytanic acid and plasmalogen for the screening of peroxisomal disorders. Brain Dev. 25, 481-487 (2003).

4 Moser, A. B., Kreiter, N., Bezman, L., Lu, S., Raymond, G. V., Naidu, S. et al. Plasma very long chain fatty acids in 3000 peroxisome disease patients and 29000 controls. Ann. Neurol. 45, 100-110 (1999).

5 Boehm, C. D., Cutting, G. R., Lachtermacher, M. B., Moser, H. W. \& Chong, S. S. Accurate DNA-based diagnostic and carrier testing for X-linked adrenoleukodystrophy. Mol. Genet. Metab. 66, 128-136 (1999).

6 Hubbard, W. C., Moser, A. B., Liu, A. C., Jones, R. O., Steinberg, S. J., Lorey, F. et al. Newborn screening for X-linked adrenoleukodystrophy (X-ALD): validation of a combined liquid chromatography-tandem mass spectrometric (LC-MS/MS) method. Mol. Genet. Metab. 97, 212-220 (2009).

7 Moser, H. W., Raymond, G. V., Lu, S. E., Muenz, L. R., Moser, A. B., Xu, J. et al. Follow-up of 89 asymptomatic patients with adrenoleukodystrophy treated with Lorenzo's oil. Arch. Neurol. 62, 1073-1080 (2005).

8 Dubey, P., Raymond, G. V., Moser, A. B., Kharkar, S., Bezman, L. \& Moser, H. W. Adrenal insufficiency in asymptomatic adrenoleukodystrophy patients identified by very long-chain fatty acid screening. J. Pediatr. 146, 528-532 (2005).

9 Takemoto, Y., Suzuki, Y., Tamakoshi, A., Onodera, O., Tsuji, S., Hashimoto, T. et al. Epidemiology of X-linked adrenoleukodystrophy in Japan. J. Hum. Genet. 47, 590-593 (2002).

10 Suzuki, Y., Takemoto, Y., Shimozawa, N., Imanaka, T., Kato, S., Furuya, H. et al. Natural history of X-linked adrenoleukodystrophy in Japan. Brain Dev. 27, 353-357 (2005).

11 Cartier, N., Hacein-Bey-Abina, S., Bartholomae, C. C., Veres, G., Schmidt, M., Kutschera, I. et al. Hematopoietic stem cell gene therapy with a lentiviral vector in X-linked adrenoleukodystrophy. Science 326, 818-823 (2009). 